\title{
ANALYSIS OF CLINICAL OUTCOMES BASED ON MOLECULAR SUBTYPES IN PATIENTS WITH BREAST CANCER THAT RECEIVED NEOADJUVANT CHEMOTHERAPY
}

\author{
Gustavo Nader Marta',2, Max S. Mano³, Leandro Jonata Oliveira3 ${ }^{3}$, Allan A. Lima Pereira³ \\ 'Department of Radiation Oncology, Hospital Sírio-Libanês - São Paulo (SP), Brazil. \\ 2Department of Radiology and Oncology, Radiation Oncology Unit, School of Medicine, Universidade de São Paulo, \\ Instituto do Câncer do Estado de São Paulo - São Paulo (SP), Brazil. \\ ${ }^{3}$ Department of Clinical Oncology, Hospital Sírio-Libanês - São Paulo (SP), Brazil.
}

Objectives: To evaluate the survival outcomes based on molecular subtypes of patients with breast cancer that received neoadjuvant chemotherapy (NAC). Methodology: We performed a retrospective analysis of all non-metastatic breast cancer patients treated between 2008 and 2014 at two institutions who had received NAC followed by surgery and postoperative radiation therapy. Patients were divided into four groups based on the tumor molecular subtype: luminal (estrogen receptor [ER] / progesterone receptor [PR] positive, human epithelial growth factor receptor-2 [HER2] negative), HER2 (HER2 positive), and triple negative (TNBC; HER2, ER and PR negative). Multivariate analyses for disease-free survival (DFS) and overall survival (OS) were also performed adjusting for unbalanced variables: pathologic complete response (pCR), histologic type and grade tumor. Results: A total of 653 women were included. Most (589; 91.1\%) of the patients had locally advanced disease (clinical stage IIB to IIIC). Patients were distributed as following (N; \%): luminal (300; 45.9\%), HER2 (173; 26.5\%), TNBC (180; 27.6\%). The groups differ regarding pathologic complete response rate (pCR), histologic type and grade, where pCR were more frequent in HER2 (64; 37.0\%) and TNBC $(52 ; 28.9 \%)$ vs. Luminal (18;6\%). The median follow-up time for surviving patients was 33 months. The disease-free survival (DFS) and overall survival (OS) rates for all patients at 3 years were 71 and 86\%, respectively. The DFS and OS rates for all patients at 3 years were 71 and $86 \%$, respectively. Patients with TNBC had worse DFS and OS rates at 3 years: (3year-DFS: 72, 76, and 64\%; 3year-OS: 88, 88 and 78\%, for Luminal, HER2 and TNBC, respectively). Comparing to TNBC, Luminal had better DFS (HR 0.67; 95\%CI 0.46-0.97) and OS (HR 0.54; 95\%CI 0.32-0.90), in multivariate analysis, while no statically difference was seen between HER2 vs. TNBC (DFS HR 0.73; 95\%CI 0.49-1.10; OS HR 0.63; 95\%CI 0.36-1.09). Conclusion: In locally advanced breast cancer patients who underwent NAC, survival rates were different based on the molecular subtype, with TNBC having the poorest prognosis. 